\title{
Stage 2 processing and the divided-attention effect
}

\author{
DOUGLAS GRIFFITH \\ Army Research Institute, Fort Hood, Texas 76544 \\ and \\ WILLIAM A. JOHNSTON \\ University of Utah, Salt Lake City, Utah 84112
}

\begin{abstract}
In two experiments subjects performed a choice-reaction-time (CRT) task and a second task concurrently. The second task was a simple reaction-time task in Experiment 1 and sentence generation and recall tasks in Experiment 2. Both studies yielded two main findings: Performance of the second task was not affected by CRT memory load (one, two, or four items), and the requirement to perform the second task did not affect the rate of increase of CRT with memory load. The first finding indicates that the effect of memory load on CRT is attributable to memory scanning, or Stage 2 processing, and is not an artifact of the rehearsal demands of the CRT memory set. The second finding indicates that Stage 2 processing is not sensitive to division of attention between the CRT and a second task.
\end{abstract}

A basic task employed in information-processing studies is the choice-reaction-time (CRT) task. Using Smith's (1968) nomenclature, a CRT task can be subdivided into four stages: Stage 1, in which the stimulus is preprocessed until an internal representation is formed; Stage 2, or memory scanning, in which the internal rep. resentation is compared with the memorial representations of stimulus alternatives; Stage 3, in which an appropriate response is selected on the basis of this categorization; and Stage 4, in which the execution of the response is programmed.

Sternberg $(1969,1975)$ has refined a methodology by which to separately analyze different stages of CRT tasks. Sternberg's item-classification task requires individuals to classify items on the basis of whether they belong to a previously memorized set of items, that is, the positive set. The research strategy, termed the "method of additive factors," is to employ factorial experiments. When factors interact, it is inferred that the factors are affecting the same processing stage. When the factors are additive, the inference is that they are affecting different processing stages. Converging operations are then employed to specify the particular stages affected. If it can be assumed that one factor affects a given stage, it is easy to ascertain whether the two factors interact. It has been assumed that the effect of memory load (ML), that is, number of items in the positive set, is localized to Stage 2. Hence, if a given factor interacts

When this research was conducted, the senior author was supported by a NIMH training grant (MH11273) awarded to the University of Utah. Requests for reprints should be sent to Douglas Griffith, Army Research Institute Field Unit, HQ TCATA, Fort Hood, Texas 76544. with ML, it would be inferred that this second factor also affects Stage 2 processing.

Another basic task employed in informationprocessing studies is the divided-attention task. Individuals are required to perform at least two component tasks concurrently in a divided-attention task. When both tasks make processing demands and when these processing demands taken together exceed the capacity available, decrements in the performance of one or both tasks inevitably result. The decrement in performance of one task can then be used to estimate the processing demands of a concurrent task (Kerr, 1973).

The present experiments examined the relationship between Stage 2 processing and the divided-attention effect. Experiment 1 used a divided-attention procedure to evaluate the assumption that the effect of ML on CRT is, in fact, localized to Stage 2 of Smith's paradigm. Upon obtaining empirical support for this assumption from Experiment 1, we carried out Experiment 2 in an effort to examine the effects of a concurrent task on Stage 2 processing when the concurrent task made heavy mnemonic demands.

\section{EXPERIMENT 1}

It is possible that the effect of ML is not localized to Stage 2 of Smith's paradigm. Rather than representing Stage 2 processing, the ML effect may simply reflect the capacity required to maintain the positive set in working memory. A reasonable assumption is that the larger the memory set, the more the capacity allocated for rehearsing the set, and the less the residual capacity available for processing the CRT stimuli. Hence, the ML effect may reflect the proportion of capacity withdrawn 
from all four stages of CRT processing and allocated to rehearsal of the positive set. Our first experiment was designed to test this alternative interpretation of the effect of ML on CRT.

The experiment was patterned after that of Wattenbarger and Pachella (1972). They reasoned that any impact of the rehearsal demands of the memory set on the processing of CRT stimuli should show up in the processing of other stimuli as well. They had subjects perform a Sternberg CRT task both by itself and concurrently with a second reaction-time (RT) task. The CRT task entailed memory sets of from one to five letters, and the second task merely required left and right buttonpresses to left- and right-pointed arrows, respectively. The dual-task requirement did not interact with ML. More importantly, the size of ML for the CRT task had no effect on the response time to the arrows. Thus, there was no indication that the rehearsal of a CRT memory set consumed capacity that could otherwise have been devoted to the processing of both CRT and second-task stimuli.

On the other hand, at least one other experiment has demonstrated that the maintenance of information in working storage retards performance of a second task. Johnston, Greenberg, Fisher, and Martin (1970) found that the performance of a subsidiary tracking task decreased monotonically as ML in a primary retention task increased from zero to eight words. The failure of Wattenbarger and Pachella (1972) to find a similar relationship between RT to arrows and CRT ML may have been an artifact of their particular procedures. Their two tasks entailed the presentation of visual stimuli at predictable points in the time from the same display location, called for identical responses, and received equal emphases when performed together. These procedural features may have encouraged subjects in the dual-task condition to adopt the strategy of keeping the positive set in a passive state of storage except when letter stimuli occurred. The subjects might have first determined whether a stimulus was an arrow or a letter and then activated the positive set only if it was a letter. Had the positive set been continuously active, as it presumably is when the CRT task is performed by itself, then it might have affected RT to the arrows.

The present experiment explored the relationship between CRT ML and performance of a simple RT task under conditions intended to functionally dissociate the two tasks and encourage the continuous activation of the CRT positive set. The key procedural modifications were that the CRT and RT stimuli were presented to different sense modalities at unpredictable points in time, that different responses were called for by the two tasks, and that the CRT task was designated as the primary task.

\section{Method}

Subjects and design. The subjects were 24 male and female subjects who participated for extra credit in undergraduate psychology courses. The overall design comprised seven treatments: the RT task alone (RT only), the CRT task alone (CRT only) with MLs of one, two, and four items, and the two tasks together (CRT-RT) with MLs of one, two, and four items. Within this overall design the dependent variables of CRT and RT defined designs of their own. The CRT task comprised a 2 by 3 factorial design in which the factors were division of attention (CRT only and CRT-RT) and ML (one, two, and four items). The RT task comprised a one-way design with CRT MLs of zero (RT only), one, two, and four. Repeated measures were taken on all factors.

Task and apparatus. The subject's cubicle was equipped with a stereo headset, a light source, and three response buttons. The subjects received instructions and CRT stimuli (letters) via the headset and RT signals via the light source. The light source was a $7.63 \times 7.63 \mathrm{~cm}$ translucent globe that was positioned at eye level about $60.96 \mathrm{~cm}$ in front of the subject. An RT signal was an increase in the intensity of the light source of $500-\mathrm{msec}$ duration. The standard intensity was $8 \mathrm{fL}$, and the signal intensity was $32 \mathrm{fL}$. The subjects used their left index fingers for the RT button and their right index and middle fingers, respectively, for the YES and NO CRT buttons.

The instructions and CRT stimuli were prerecorded on the right channel of a stereo tape recorder and played to the subjects binaurally at $65 \mathrm{~dB}$. A CRT stimulus closed a sound-sensitive relay and thereby started a millisecond timer. The timer was stopped when the subject pushed a CRT button. The RT signals were triggered by prerecorded tones from the left channel (which was not played to the subjects) of the tape recorder. A tone closed a second sound-sensitive relay and thereby both triggered an RT signal and started a second millisecond timer. The second timer was stopped when the subject pushed the RT button. The subjects' CRTs and RTs were recorded by an experimenter who was stationed outside the subjects' cubicle.

Procedure. Each subject served for seven consecutive 5-min trials in a single session. A 30 -sec break was provided between trials. The first trial was always an RT-only trial. The six remaining trials were three CRT trials followed by three CRT-RT trials for half the subjects, and vice versa for the other half of the subjects. The order of the three CRT memory loads within each block of three trials was counterbalanced across subjects. The subjects were instructed to regard the CRT component of CRTRT trials as primary and the RT component as secondary.

Stimulus programming. A stimulus (light signal or letter) was programmed into every 10 -sec interval of each trial. The localization of stimuli within these intervals was random, with the restriction that no two stimuli could occur within $3 \mathrm{sec}$ of one another. The CRT stimuli were the letters A, B, F, G, I, K, L, $\mathrm{N}, \mathrm{O}, \mathrm{P}, \mathrm{Q}, \mathrm{R}, \mathrm{U}$, and $\mathrm{Y}$. The memory set was announced $15 \mathrm{sec}$ in advance of each trial involving the CRT task. Each of the 14 letters was used precisely once in composing the memory sets for a subject. The assignment of letters to the $10-\mathrm{sec}$ intervals of a trial was random except that the probability of a memory set letter had to be .50 and that, across subjects and trials, the frequency of occurrence of individual letters had to be as constant as possible. Across subjects, the exact sequence of CRT stimuli was held constant between the CRT-only and CRT-RT conditions. That is, the sequence of letters received by one subject under a given $\mathrm{ML}$ in the CRT-only condition was received by another subject under that same ML in the CRT-RT condition. In all, each subject produced 30 RTs under each condition involving the RT task and 30 CRTs under each condition involving the CRT task.

\section{Results and Discussion}

Reaction time. The focus of the present study was on the possible effect of CRT ML on simple RT. A perusal 
of the left-hand panel of Figure 1 reveals that, although the requirement of performing the CRT task increased RT, RT was not a function of the size of the ML. Analyses of variance and Newman-Keuls tests performed on the number of missed signals and on the mean RT confirmed the conclusion that the rehearsal demands in the typical Sternberg item-recognition task are negligible. ${ }^{1}$ The alpha level was set at .05 for all tests. Although the effect of ML in the analysis of variance of number of missed signals was significant $[F(3,69)=$ $11.45, \mathrm{MSe}=8.37 \mathrm{]}$, a Newman-Keuls test localized the effect solely to the increase in missed signals between RT-only and dual-task conditions. The mean number of missed RT signals (maximum $=30.0$ ) was $1.25,5.17$, 4.13, and 5.75 for CRT MLs of $0,1,2$, and 4, respectively. The effect of ML was also significant with respect to simple $\operatorname{RT}[F(3,69)=65.17$, MSe $=2254]$, but again was due strictly to the increase in simple RT between RT-only and the dual-task conditions. Hence, the effect of ML on CRT is not mirrored by simple RT.

Choice reaction time. The CRT data are summarized in the right-hand panel of Figure 1. These data confirm the conclusion reached by others (e.g., Briggs, Peters, \& Fisher, 1972; Wattenbarger \& Pachella, 1972) that the dual-task requirement does not interact with ML. An analysis of variance of correct CRT validated this conclusion. Whereas the main effects of division of attention $[\mathrm{F}(2,46)=22.79$, MSe $=4,440]$ and memory load $[\mathrm{F}(2,46)=76.52, \mathrm{MSe}=5,025]$ were significant, their interaction was not significant $[\mathrm{F}(2,46)=1.70, \mathrm{MSe}=$ $6,776]$. Only $5.4 \%$ of the CRT stimuli were misclassified, and the frequency of these response errors was constant across conditions (all Fs $<2.71$ ).

The observed insensitivity of Stage 2 processing to division of attention in this and prior experiments (Briggs et al., 1972; Wattenbarger \& Pachella, 1972) may be attributable to the nature of the second task. Perhaps if the secondary task had received more emphasis and placed heavier demands on mnemonic processing, it would have affected Stage 2 memory scanning with

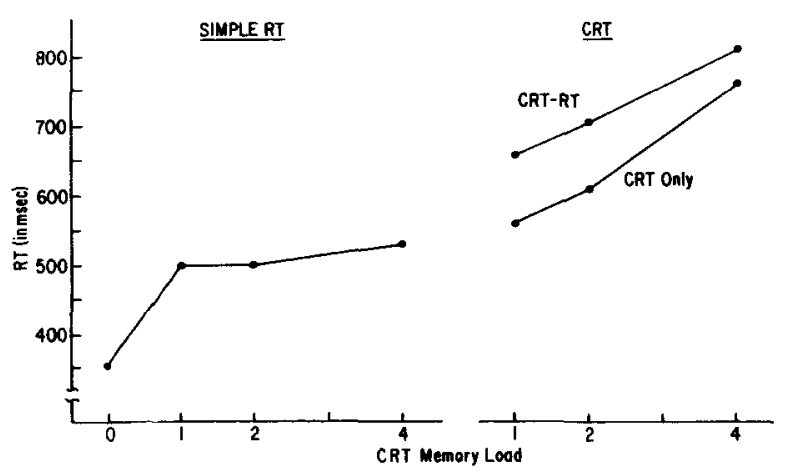

Figure 1. Simple RT (left panel) as a function of CRT memory load and CRT (right panel) as a function of memory load and division of attention. respect to the CRT task. Experiment 2 was designed to assess this probability.

\section{EXPERIMENT 2}

Briggs et al. (1972) used the Sternberg procedure in an effort to pinpoint the processing locus of the dividedattention effect, that is, the decline in the performance of one task owing to the requirement to perform a second task concurrently. They had subjects perform a CRT task and a pursuit tracking task simultaneously. The dual-task requirement did not interact with $M L$, indicating that Stage 2 was not the locus of the dividedattention effect. Through the use of converging operations, Briggs et al. attributed the divided-attention effect to Stage 1 as opposed to Stage 3 or 4 .

Having vindicated the diagnostic logic employed by Briggs et al. (1972) in Experiment 1, we sought next to assess the generality of their findings. As Briggs et al. pointed out, it is possible that a second task that places a relatively heavy load on memory processes could affect Stage 2 processsing. We ran Experiment 2 in an effort to test this possibility. Two tasks were chosen which were thought to make relatively heavy demands on memory: a sentence-generation task and a recall task. Subjects were required first to form a sentence mediator for each of several noun pairs and then to recall the second noun of each pair upon receiving the first noun as a cue. It was expected that at least one of the tasks would affect Stage 2 processing with respect to a CRT task. In order to increase the sensitivity of CRT to the division-of-attention requirement, the subjects were explicitly instructed to regard the sentence-generation and recall tasks as primary and the CRT task as secondary.

\section{Method}

Subjects and design. The subjects were 18 male and female students who received extra credit in an introductory psychology class in return for their service. The basic design was a 3 by 3 factorial with repeated measures on both factors. The two factors were division of attention (CRT only, sentence generation CRT, and recall-CRT) and ML (one, two, and four). Each subject also performed under a memory-only control condition in which the sentence-generation and recall tasks were performed by themselves.

Tasks and apparatus. The subject cubicle was equipped with a stereo headset and two response buttons labeled YES and NO. The right channel of the headset was used for the memory task, and the left channel for the CRT task. The sound level on both channels was around $65 \mathrm{~dB}$. The subjects rested their right and left index fingers on the YES and NO buttons, respectively. The stimulus information for the two tasks was played to subjects from a stereo tape recorder located at an experimenter station outside of the subject cubicle. The left channel of the tape recorder was linked to a millisecond timer by way of a voice key. A CRT stimulus started the timer, and the subject's keypress response stopped it. The subject's verbal responses in the memory task were recorded on a second tape recorder and later scored for accuracy.

The sentence-generation task required subjects to produce a sentence of the basic form "article Noun 1 verbed article Noun 2" 
for each of 32 noun pairs. The noun pairs were presented at a 10-sec rate: $2 \mathrm{sec}$ for the presentation of Noun 1/Noun 2 (e.g., STORM-HAMLET), $5 \mathrm{sec}$ for covert sentence generation, and $3 \mathrm{sec}$ for the overt production of the sentence (e.g., "THE STORM WRECKED THE HAMLET"). The production period was signaled by a tone.

The recall task always followed the sentence-generation task and proceeded at the same rate: $2 \mathrm{sec}$ for the presentation of the word "recall" and Noun 1 (e.g., RECALL, STORM), $5 \mathrm{sec}$ for the covert retrieval of Noun 2, and $3 \mathrm{sec}$ for the overt emission of Noun 2 (e.g., HAMLET). The overt recall period was signaled by a tone. When the CRT task was performed concurrently with the memory task, a CRT stimulus always occurred at one of the first $4 \mathrm{sec}$ (stimulus loci) of the covert generation or retrieval period. The CRT stimuli occurred at the same rate when the CRT task was performed by itself (CRT only): The words READY START occurred every $10 \mathrm{sec}$ and were followed, at one of the four stimulus loci, by a CRT stimulus. Each of the 10 -sec intervals comprising a task will be referred to henceforth as a "trial."

Procedure. Following practice at performing the memory and CRT tasks, both separately and concurrently, the subjects performed four trial blocks. The practice trials and the first two trial blocks were administered in one session, and the last two trial blocks were administered in a second session $24 \mathrm{~h}$ later. Each trial block involved a different list of 32 noun pairs. Half of the subjects received the four lists in one order and half in the reverse order. The memory-only condition was administered on the first trial block and the nine conditions of the basic design were administered over the last three trial blocks. Each of the last three trial blocks was represented by a different level of ML and was composed of, in order, 16 CRT-only trials, 32 sentence-generation/CRT trials, 32 recall-CRT trials, and a final 16 CRT-only trials. The memory set for the CRT task was announced immediately prior to each trial block. Thus, the three levels of division of attention were manipulated within blocks, and the three levels of ML were manipulated across blocks. The interblock order of the three MLs was counterbalanced across subjects and list orders. In all, each subject performed 32 trials under each condition of the experiment.

On dual-task trials, subjects were instructed to regard the memory task as primary and the CRT task as secondary. They were asked to devote as much attention to the memory task as was necessary to perform it at a maximal level and to devote all of their leftover attention to the CRT task.

Stimulus materials. The memory lists were constructed using high-imagery nouns (all imagery values $>4.00$ ) drawn from the Paivio, Yuille, and Madigan norms (1968). The noun pairs were systematicaily constructed so that each lent itself to the generation of a simple and sensible sentence. The recall order of the noun pairs for each list was random, with the restriction that each pair be tested exactly once.

The CRT stimuli were the eight monosyllabic digits 1 through 9, excluding 7 . The digits were assigned to the three memory sets (Loads 1,2 , and 4) randomly without replacement. The stimulus digits for the 32 trials under a given condition (e.g., CRT only, ML 1) were determined randomly with two restrictions: that the probability of a memory set digit be .50 and that the four stimulus loci be used equally often.

\section{Results and Discussion}

The mean correct CRT data (equally weighted for positive and negative instances) are plotted as a function of ML in Figure 2. An analysis of variance (alpha was set at .05 for all statistical tests) indicated that, although the main effects were significant $[F(2,34)=43.68$, MSe $=2,140$ for division of attention and $F(2,34)=$

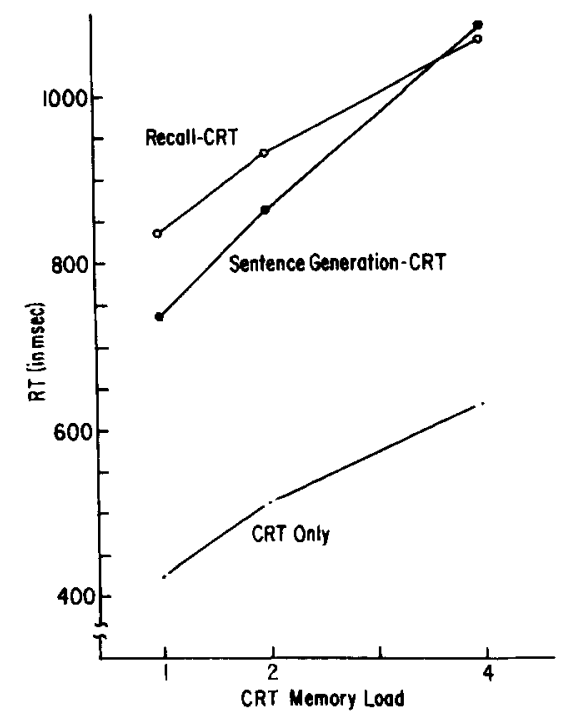

Figure 2. Choice RT as a function of memory load for a single-task condition (CRT only) and two dual-task conditions (sentence generation/CRT and recall-CRT).

$58.48, \mathrm{MSe}=4,888$ for $\mathrm{ML}]$, the interaction was not significant $[F(4,68)=2.06, \mathrm{MSe}=1,254]$. Thus, the apparent increase in slope between CRT-only and the dual-task conditions was not statistically reliable and, in any case, was minute in comparison with the massive increase in the intercept. Likewise, the slope and intercept differences between the two dual-task conditions were relatively small, statistically nonsignificant, and of little interest in the present context.

Ancillary analyses were performed on CRT accuracy and recall accuracy. About $8 \%$ of the CRT stimuli were misclassified, and the frequency of these response errors increased with ML $[F(2,34)=10.26, \mathrm{MSe}=.84]$. The mean number of words correctly recalled under CRT MLs of 0 (memory only), 1, 2, and 4 was $22.38,20.00$, 19.61 , and 20.33 , respectively. Although the effect of ML was significant $[\mathrm{F}(3,51)=3.25, \mathrm{MSe}=8.48]$, a Newman-Keuls test localized the effect to the higher level of recall in the memory-only than in the dual-task conditions. Within the dual-task conditions, the level of ML did not affect recall performance.

The key finding in the above data is the absence of a significant interaction between ML and the division-ofattention requirement. This finding substantiates further the conclusion that Stage 2 is not affected by division of attention. Apparently the memory scan occurs automatically regardless of the specific requirements of additional tasks. In contrast to the perceptual-motor task employed by Briggs et al. (1972), the sentencegeneration and recall tasks should make heavy mnemonic demands on, and should be most likely to tap into, the Stage 2 processing machinery. However, it is the absolute magnitude of the intercept effect, and not the 
slope, which more than doubled that in the Briggs et al. study. It appears, then, that Stage 2 is relatively impervious to additional processing demands.

\section{GENERAL DISCUSSION}

We submit the following two conclusions based on the data of the present and prior (e.g., Briggs et al., 1972; Wattenbarger \& Pachella, 1972) studies: (1) When the size of the CRT ML is within the immediate memory span, the performance of a second task will not be affected by ML; (2) Stage 2 processing, as indexed by the effect of ML on CRT, is unaffected by the processing demands of a second task. These two conclusions must be considered to have a high degree of generality, as they have withstood wide variations in the nature of the second task and in the emphasis placed on the second task relative to the CRT task: tracking, equal emphasis (Briggs et al., 1972); two-choice RT, equal emphasis (Wattenbarger \& Pachella, 1972); simple RT, secondary emphasis (Experiment 1); sentence generation, primary emphasis (Experiment 2); and recall, primary emphasis (Experiment 2). The first conclusion indicates that the effect of ML on CRT is, indeed, localized to Stage 2 of Smith's paradigm. The second conclusion indicates that Stage 2 processing is automatic, that is, the memory scan is conducted without interruption even when a second task is being performed at the same time.

\section{REFERENCES}

Briggs, G. E., Peters, G. L., \& Fisher, R. P. On the locus of divided attention effects. Perception \& Psychophysics, 1972, 11, 315-320.

Johnston, W. A., Greenberg, S. W., Fisher, R. P., \& Martin, D. W. Divided attention: A vehicle for monitoring memory processes. Journal of Experimental Psychology, 1970, 83, 164-171.

KerR, B. Processing demands during mental operations. Memory \& Cognition, 1973, 1, 401-412.

Patvio, A., Yuille, J. C., \& Madigan, S. Concreteness, imagery, and meaningfulness values for 925 nouns. Journal of Experimental Psychology Monograph Supplement, 1968, 76(1, Part 2).

SMTrH, E. E. Choice reaction time: An analysis of the major theoretical positions. Psychological Bulletin, 1968, 69, 77-110.

SternBerg, S. Memory scanning: Mental processes revealed by reaction-time experiments. American Scientist; 1969, 57, 421-457.

Sterngerg, S. Memory scanning: New findings and current controversies. Quarterly Joumal of Experimental Psychology, 1975, 27, 1-32.

Wattenbarger, B. L., \& Pachella, R. G. The effect of memory load on reaction time in character classification. Perception \& Psychophysics, 1972. 12, 100-102.

\section{NOTE}

1. A missed signal was one to which a response did not occur within 1,200 msec. Inasmuch as less than $1 \%$ of the RTs for hits fell between 800 and $1,200 \mathrm{msec}$, this cutoff point was considered appropriate.

(Received for publication March 7, 1977; accepted June 2, 1977.) 